\title{
Is amblyopia due to inappropriate stimulation of the "sustained" pathway during development?
}

\author{
HISAKO IKEDA AND M. J. WRIGHT \\ Vision Research Unit, Sherrington School of Physiology, St. Thomas's Hospital Medical School, London
}

First, we should like to summarize our neurophysiological investigations on the retina and the visual cortex in the cat which are relevant to the problems of amblyopia and then to discuss a hypothesis which is slightly different from that currently accepted.

There are two aspects to amblyopia: one is a reduction of visual acuity of the fovea and the other is a disturbance of binocular vision. Our concern here is particularly with the first aspect, i.e. a reduction of visual acuity of the fovea in strabismus and related conditions.

Visual acuity is known to be closely associated with the density distribution of cone receptors throughout the retina. As the upper graph in Fig. I (overleaf) shows (redrawn from Steinberg, Reid, and Lacy, I973), the cone density is highest at the fovea and becomes sharply reduced towards the periphery of the retina for both human and cat, although the actual numbers of neurones differ considerably between the human and the cat. The lower graph of Fig. I (redrawn from the original work of Stone, I965) shows the density and size distribution of the retinal ganglion cells in the cat retina.

The ganglion cells are closely packed, being 6,000 cells $/ \mathrm{mm} . .^{2}$ at the area centralis, the equivalent point in the cat retina to the fovea of the human retina, but fall off sharply to 500 cells $/ \mathrm{mm} .^{2}$ within $5^{\circ}$ from the area centralis. Naturally the higher the density, the smaller the diameter of the cell. The cells in or near the area centralis are small, $8 \mu$ in diameter, but the peripheral cells are as large as $25 \mu$ in diameter. .

Our question was, do the properties of small central cells and large peripheral cells differ?

To study retinal ganglion cells, we used an intraretinal microelectrode introduced into the eye. We could aim at the area centralis or the peripheral retina according to our choice, using our specially designed microelectrode advancer (Ikeda and Pringle, I97 I), and then investigate individual cells using a wide range of stimulus intensity, size, duration, and location. We found many striking differences between the properties of retinal ganglion cells in or near the area centralis and those of the peripheral retina. For example, the patterns of firing of most cells in or near the area centralis and that of many peripheral cells differ in their response to a stationary spot at the receptive field centre (Fig. 2, overleaf).

Fig. 2 presents two post-stimulus histograms, showing the pattern of firing of a neurone to stimulation, obtained from an on-centre cell $20^{\circ}$ away from the area centralis of the retina (upper histogram), and from an on-centre cell in the area centralis (lower histogram). The stimulus was a small spot ( $25^{\prime}$ of arc) located at the receptive field centre and was "on" for $20 \mathrm{sec}$. and "off" for $20 \mathrm{sec}$. The peripheral cell shows a transient response when the spot appears, whereas the cell in the area centralis shows sustained excitation while the 
FIG. 2

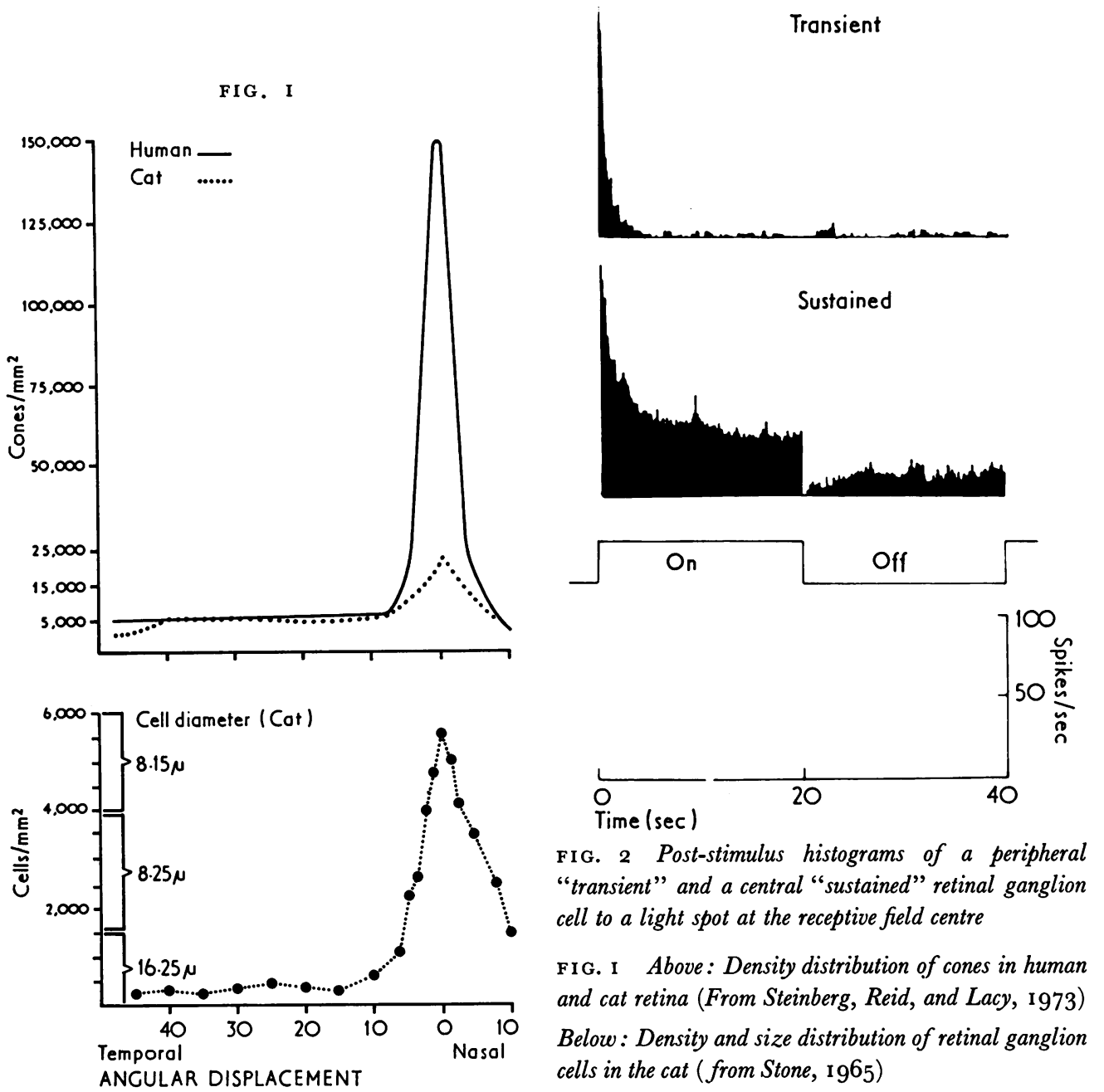

stimulus is on. It has been established that the retinal ganglion cells of the cat can be classified into two types, those which respond to a stationary contrast at the receptive field centre with transient firing, called "transient" cells (or Y-cells), and those which respond with sustained firing, called "sustained" cells (or X-cells) (Enroth-Cugell and Robson, I966; Cleland, Dubin, and Levick, I97 I ; Ikeda and Wright, I972a). "Sustained" neurones are most frequently found in or near the area centralis, while "transient" neurones are commonly found in the peripheral retina. The two groups of cells show differences in their receptive field organization, suggesting their different functional roles (Ikeda and Wright, I972a).

Fig. 3 illustrates the difference in the sensitivity gradient of "sustained" cells found in or near the area centralis and that of "transient" cells commonly found in the peripheral retina. The sensitivity gradient is a plot of the reciprocal of threshold intensity of a spot stimulus against position in the receptive field.

Fig. 3 shows that the "sustained" cell has an extremely high sensitivity at the receptive field centre, which drops sharply to zero within $0.2^{\circ}$ to $0.3^{\circ}$ from the centre. In other 


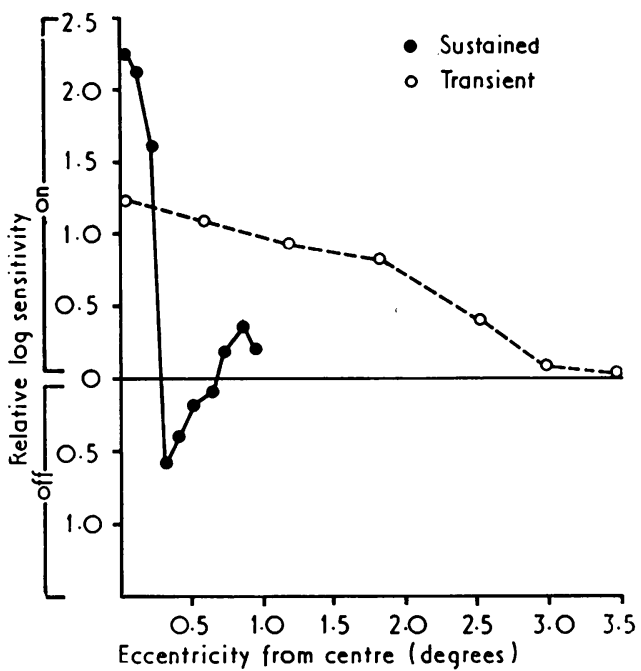

FIG. 3

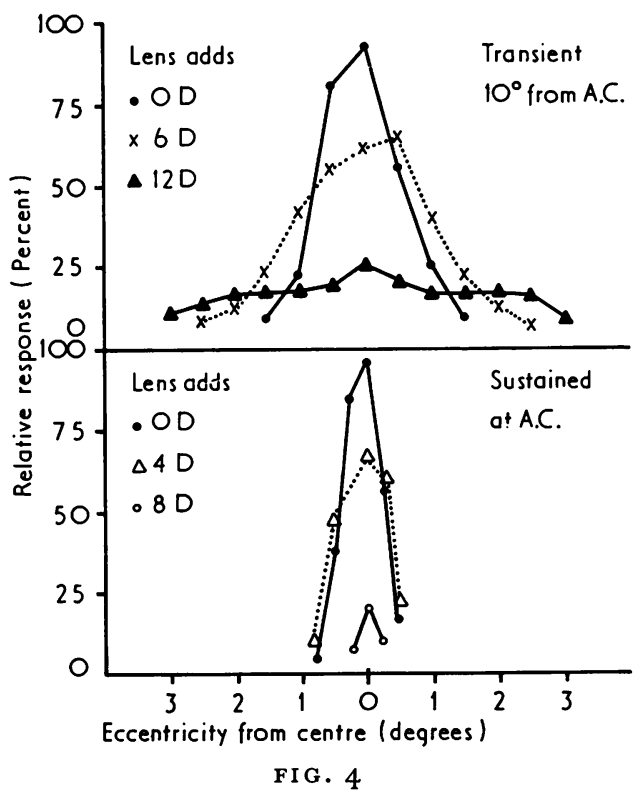

F IG. 3 Sensitivity gradients of a peripheral "transient" and a central "sustained" retinal ganglion cell across the radius of the receptive field

FIG. 4 Effect of defocusing on response profiles of a peripheral "transient" and a central "sustained" retina ganglion cell

words, the cell is capable of accurately distinguishing a minute displacement in relation to the receptive field centre. The "sustained" cell has also a well-defined "inhibitory" surround where the cell becomes more sensitive to stimulus "off" than to stimulus "on", and it then enters another outer zone called "disinhibitory" where the cell becomes again sensitive to stimulus "on" (Ikeda and Wright, I972b).

Thus "sustained" cells in or near the area centralis have (I) extremely small receptive field centres, (2) steep sensitivity gradients, and (3) well-defined surrounds; all these properties give the receptive field a capacity for fine spatial discrimination and thus provide a basis for visual acuity. However, such "sustained" neurones respond very poorly to large, low-contrast stimuli because these stimuli invade both the excitatory and the inhibitory regions of the receptive field. On the other hand, the "transient" cell has a lower sensitivity at the receptive field centre than the "sustained" cell and the sensitivity gradient is shallow, having gentle slope changes; also it never drops to zero and never exhibits a distinctive surround region. Peripheral "transient" cells have large receptive field centres and weak surrounds; they are thus poor spatial discriminators. But the "transient" peripheral neurones are very sensitive to a large object of high contrast fluctuating in time (Ikeda and Wright, 1972c).

An important difference between "sustained" central and "transient" peripheral cells which bears on the problem of amblyopia is their response to defocused stimuli (Ikeda and Wright, 1972a). This is illustrated in Fig. 4.

Each curve in Fig. 4 represents the response profile of the cell in different focusing conditions. The upper graph shows the behaviour of a peripheral "transient" cell, and the lower that of a central "sustained" cell. First look at the "transient" cell. The most sharplypeaked curve is obtained under the best focused condition. When the eyes are defocused 
progressively by lenses, the rate of firing declines at the centre. With further defocusing, the activity of the field edges becomes greater than in the focused condition. Eventually, the cell responds equally to a stimulus centred anywhere in the receptive field. So in "transient" cells, the role of the cell, under defocused conditions, changes to a light-catching device from a sharp centre-favouring one, and thus the cell is capable of responding "transiently" to a stimulus spot defocused by $12 \mathrm{D}$ or more.

In contrast, the central "sustained" cell behaves quite differently. The sharply-peaked curve is obtained in the best focused condition. Defocusing does not result in expansion of the receptive field, but the cell retains a symmetrical response profile to the point where it can no longer respond. The response of "sustained" cells can be abolished altogether by a smaller error of refraction (usually less than $8 \mathrm{D}$ ). In other words, spatially-spread, lowcontrast stimuli do not provide adequate stimulation for the central "sustained" retinal ganglion cells. It appears therefore that the fine spatial discrimination characteristic of high visual acuity is dependent on "sustained" retinal ganglion cells in the area centralis which require a sharply focused, small object as appropriate stimulus.

Furthermore, there is evidence that the information arising from the central "sustained" retinal ganglion cells and that from the peripheral "transient" ganglion cells remains separate throughout the higher visual nervous system (Fukada, I97 I C Cleland and others, I97I ; Ikeda and Wright, I974; Hoffmann. Stone, and Sherman, I972; Stone and Dreher, I974).

Recently we have shown that there are also two groups of visual cortical neurones in Area I 7 (Ikeda and Wright, I974). One group of neurones particularly receiving the projection from the area centralis responded to a stationary bar of correct orientation and width with "sustained" firing. These "sustained" firing cortical neurones are tuned to a higher spatial frequency, i.e. they respond best to fine gratings just as the "sustained" retinal ganglion cells respond to a small spot. We also found that, in order to obtain acute responses from these "sustained" neurones with high spatial frequency tuning, that we had to pay particular attention to the refractive state of the eye and centring of the artificial pupil with the visual axis which we used for the experiments on the visual cortical neurones.

The other group of cortical neurones, on the other hand, responded with "transient" firing to a stationary bar, and they had tuning to a lower spatial but higher temporal frequency, again reflecting the properties of the "transient" retinal ganglion cells (Fig. 5, opposite).

Fig. 5 shows the post-stimulus histograms of a "transient" (left) and a "sustained" (right) cortical neurone to gratings drifting across the receptive field at different speeds.

The "transient" neurone, receiving a projection from a retinal region approximately $5^{\circ}$ away from the area centralis had a preferred orientation of $225^{\circ}$ and responded best to a grating of 0.3 cycles per degree, which is a large grating. The cell responded with transient firing and was capable of responding to such a grating drifting very fast in the receptive field. As the post-stimulus histogram obtained at the drift speed of $16 \mathrm{~Hz}$. shows, the cell still responds to each bar cycle of grating when the large grating drifted across at i 6 cycles grating per second.

The "sustained" cortical neurone, having a projection from the area centralis, with orientation tuned to $180^{\circ}$ (horizontal), responded best to a grating of $\mathrm{I} \cdot 4$ cycles per degree, which is a fine grating. As the post-stimulus histogram on the right in Fig. 5 shows, the cell responded with a strong sustained firing to such a fine grating only when the grating was drifting at a very slow speed $(0.25$ or $0.5 \mathrm{~Hz}$.). The cell, however, was incapable of following each bar of such grating when the drift speed was increased to I $\mathrm{Hz}$. or $4 \mathrm{~Hz}$, , 


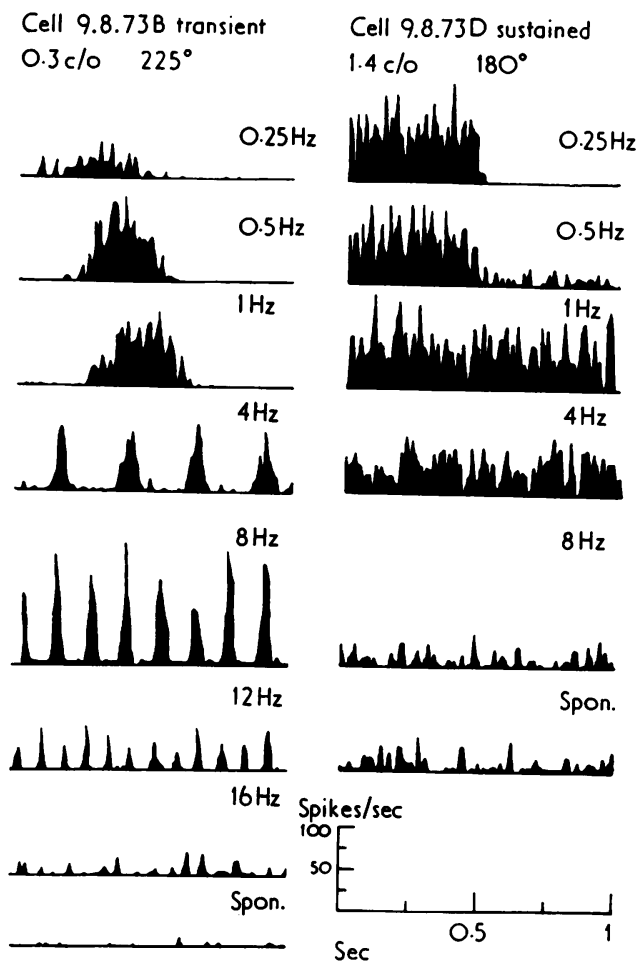

FIG. 5 Post-stimulus histograms of a "transient" and "sustained" cortical neurone in Area I7 of the cat showing the cell response to an optimal grating drifting across the receptive field at different speeds

though the general firing rate of the cell remained higher than the spontaneous level. The response of the cell to the grating drifting at $8 \mathrm{~Hz}$. is exactly the same as spontaneous firing.

It appears therefore that the "sustained" cortical neurone requires not only a sharply focused image but a temporally stable one.

In Fig. 6 we have simplified and summarized what is known of the "sustained" and

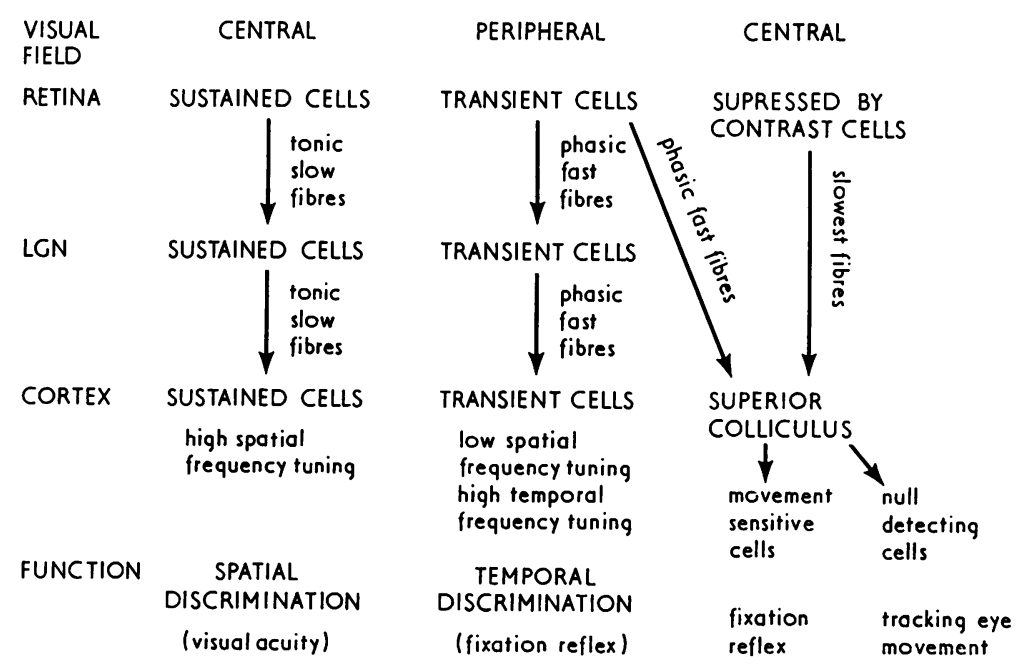

F I G. 6 Flow diagram showing "sustained" and "transient" pathways in the visual nervous system. The likelihood of the receptive field position in the visual space and possible functional roles for each pathway are also indicated 
"transient" pathways in the brain (Cleland and others, I97 I Fukada, I97 I ; Ikeda and Wright, I972C; Hoffman and others, 1972; Stone and Dreher, I973; Ikeda and Wright, I974; Hoffman, 1973).

Fig. 6 indicates that there appear to be at least two separate channels in the visual nervous system. One arises from "sustained" retinal ganglion cells and terminates in "sustained" LGN, and finally in "sustained" cortical neurones which are tuned to high spatial frequency. This pathway consists of slow-conducting fibres. The other arises from "transient" retinal ganglion cells and branches out into two; one branch goes to the superior colliculus which is involved in fixation reflex and eye movements, and the other branch terminates in "transient" LGN neurones, and finally in "transient" cortical neurones which have low spatial but high temporal frequency tuning. This pathway consists of fastconducting fibres.

In addition, there is a third class of retinal ganglion cells called "suppressed by contrast" or "W"-cells, which have small receptive fields and terminate exclusively in the superior colliculus. This pathways consists of the slowest-conducting optic nerve fibres.

It is reasonable to suggest that the "sustained" neurones are those which provide fine spatial discrimination and are thus the basis for high visual acuity, while the "transient" neurones are those which provide the basis for analysis of movement and initiation of visual fixation reflexes. The fact that the retinal ganglion cells also terminate in the superior colliculus supports the idea that the "transient" cells respond to "attention-getting" stimuli in the peripheral field. Furthermore, the least documented "suppressed by contrast" cells from the area centralis of the retina terminating exclusively in the superior colliculus may be involved in control of slow tracking eye movement or maintenance of fixation, and thus may also function abnormally in strabismus; this, however, is not our topic for discussion, since we are primarily concerned with the reduction of visual acuity in strabismus.

From these considerations and the fact that amblyopia develops in early life with a reduction of the visual acuity of the fovea, we tentatively suggest that, in cases of amblyopia, "sustained" neurones in the retina have been deprived of adequate stimulation during early development, so that the "sustained" pathway has become ineffective or has degenerated.

In order to explain this suggestion more closely in the clinical situation, we will consider Fig. 7 (opposite), which is a simplified diagram of the nervous system in normal and squint amblyopia.

As the diagram on the left of Fig. 7 illustrates, healthy eyes are designed to form a clear image of an object of interest, sharply focused on the fovea of both eyes by means of a fixation reflex, convergence, and accommodation. The fibres of the foveal retinal ganglion cells, presumably "sustained" cells from both eyes, terminate in a column, in alternate laminae in the LGN. The fibres from these LGN neurones meet at the visual cortex. The cortical neurones of high spatial tuning may thus be driven by stimulation of either fovea.

In strabismus, though clinical variations are immense, the eyes all have in common a situation like that shown in the diagram on the right of Fig. 7. The clearly focused image of this object is formed on the normal eye, but the fovea of the deviating eye sees some other object which may or may not lie at the same distance as the object of interest. The object of interest is seen by the squinting eye at some peripheral part of the retina. But the peripheral retinal neurones terminate in a different part of the LGN and also in a different part of the cortex.

The fibres from the fovea of the normal eye carrying information about the object of interest, and those from the fovea of the squinting eye carrying information about some 


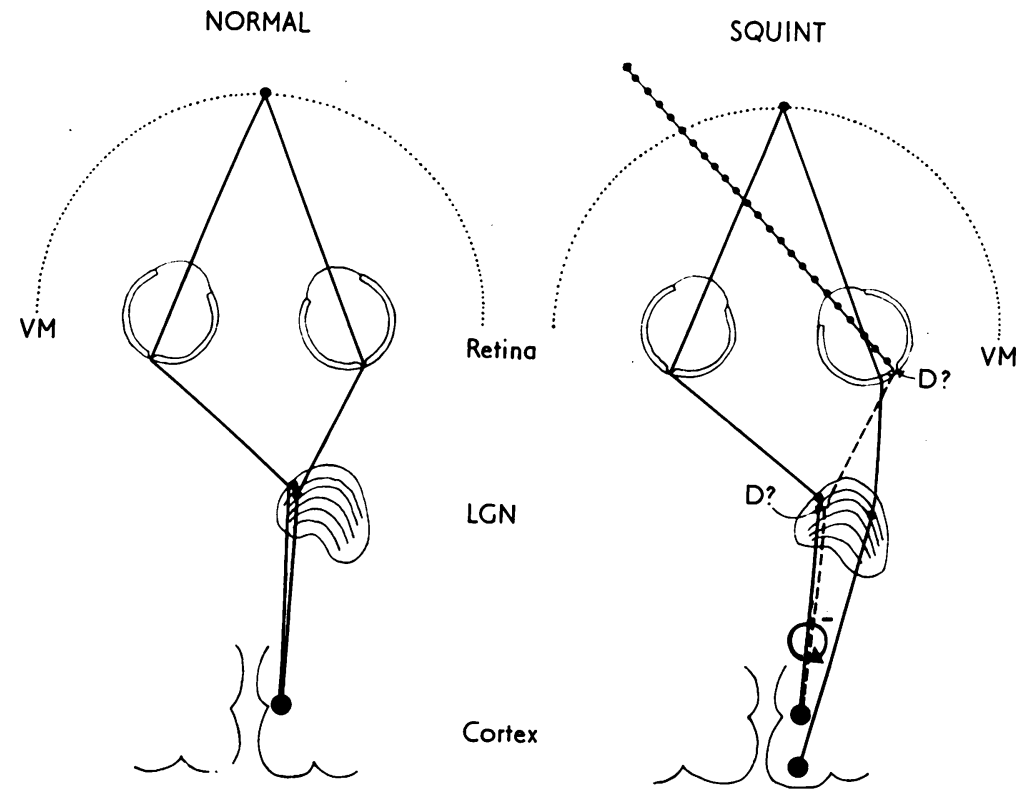

FIG. 7 Simplified diagrams of visual pathways in cases of normal and squint amblyopia. Solid line indicates normal path and dotted line a possible degenerative path. The curved arrow with minus sign indicates the cortical suppression theory

other uninteresting object, often defocused, project to a single column, but to different laminae of the column in the LGN, and finally meet in the cortex.

Now the classical theory of amblyopia is that the neural message from the fovea of the squinting eye is suppressed in the same way as in the normal subject (i.e. when the two eyes receive dissimilar stimulation, one image is suppressed), and that this suppression becomes permanently established. However, there is no neurophysiological evidence that the strong unwanted image from the squinting eye is suppressed at the cortex.

In amblyopia the change we have to look for is a subtle one, in which the foveal visual acuity is reduced from $6 / 6$ to $6 / 18$ or $6 / 36$, but flicker response to flash or colour vision and other visual functions are unaffected (Duke-Elder and Abrams, 1970). Such subtle changes may be studied only when the neurones in the visual system are examined very thoroughly by measuring sensitivity, and such neurophysiological experiments have not yet been reported.

However, there is a great deal of evidence that abnormal visual experience during a sensitive period of development produces near-permanent physiological abnormalities in the visual nervous system (Wiesel and Hubel, 1963; von Noorden and Dowling, 1970; Blakemore and Cooper, I970; Freeman, Mitchell, and Millodot, 1972). From the simple hypothesis of Wiesel and Hubel ( 1963$)$, that a neural connection requires adequate stimulation at a sensitive period of development in order to remain effective, we considered whether the "sustained" retinal ganglion cells of the fovea of squinting eyes might already be modified by inadequate stimulation during early development, so that the "sustained" pathway arising from the squinting fovea becomes ineffective with inadequate synaptic connections, so reducing visual acuity.

We have shown that the "sustained" retinal ganglion cells in the fovea require well focused and small stimuli in order to respond effectively. If, however, the foveal retinal 
ganglion cells of the squinting eye were deprived of finely focused stimuli, particularly of close objects, this ineffective stimulation could produce a failure of development in the neurones which provide fine spatial discrimination. Thus amblyopia-reduction of visual acuity-may result from lack of adequate stimulation of the fovea rather than from an active suppression of the strong input from the squinting eye.

If we look at other forms of amblyopia, this seems more likely.

Fig. 8 illustrates the situation of myopia, hypermetropia, and anisometropia. In congenital myopia, amblyopia is uncommon (Duke-Elder and Abrams, 1970), presumably because, as the left diagram of Fig. 8 illustrates, a myopic person sees an object of interest very close to the eye, clearly focused on the fovea of both eyes. Thus the fovea is never completely deprived of correct stimulation. The foveal "sustained" retinal ganglion cells, LGN cells, and cortical neurones are thus activated normally. After refractive correction the visual acuity is normal.
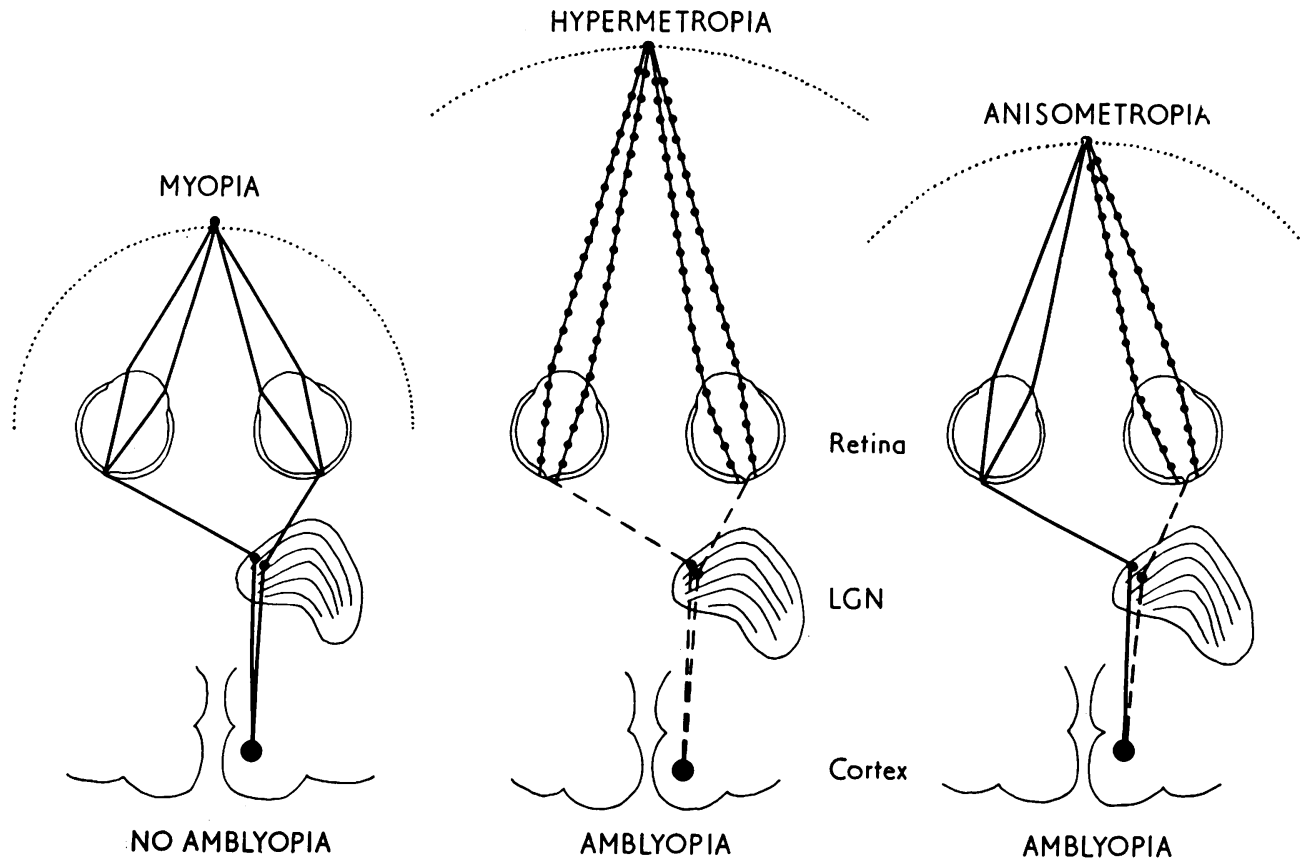

FI G. 8 Simplified diagrams of visual pathways in cases of congenital myopia, hypermetropia, and anisometropia. The dotted lines indicate possible degenerative paths

The middle diagram shows a case of severe congenital hypermetropia. This is clinically uncommon, as in such cases a squint may occur to accept a better image. However, we have learnt from Mr. Peter Fells (personal communication) that, if both eyes are grossly hypermetropic, a child may learn to accept a blurred image without producing squint or diplopia. In such cases, amblyopia follows, in spite of the fact that the nervous connections are the same as those in normal and myopic persons. This is what we should expect if our hypothesis is correct.

The diagram on the right in Fig. 8 is a clinically common case of anisometropia. Again a squint often occurs, but even in cases in which no squint is present, a hypermetropic or astigmatic eye becomes amblyopic if the fovea receives blurred images continuously during 
early development. Thus, once again, it appears that, when the fovea is deprived of clearly focused image stimulation, the result is amblyopia.

To summarize our argument:

(I) Foveal "sustained" neurones in the retina which provide the basis for fine spatial discrimination and visual acuity, require well focused stimuli in order to respond effectively.

(2) Neural connections require appropriate stimulation during a sensitive period of development if they are to be effective in the adult.

(3) Clinically, when the fovea is deprived of clearly focused image stimulation during early development, foveal visual acuity appears to be permanently reduced. We therefore suggest that amblyopia may result in cases in which the foveal "sustained" neurones do not receive adequate stimulation at a sensitive period of development, and a functional degeneration in the sustained pathways could theoretically occur as early as the retinal ganglion cells.

We thank the M.R.G. and the R.N.I.B. for their financial support for the research, and also Miss Janet Nuza, Mr. J. Dench, and Mr. A. Allchurch for their technical assistance.

\section{Discussion}

WATSON Is there any histological evidence for the changes which you have described?

IKEDA No, there is no evidence because unfortunately there was an acute epidemic in the cat colony and all the cats with surgically-produced squint were lost, so that the neurophysiological and histological examinations of these important animals were never completed. But in several behavioural tests these cats showed some evidence of a reduction of visual acuity in the squinting eye.*

VON NOORDEN We have looked at the retinal ganglion cells in the monkey with the electron microscope and have not found any degeneration. We have not been able to confirm the findings of Chow, Riesen, and Newel (1957), who said that the ganglion cells disappeared after visual deprivation. As far as we can tell the retina remained histologically normal in all cases of amblyopia.

IKEDA Have you examined the peripheral and central retina separately? The change in the ganglion cell may only occur in the small densely packed neurones in the area centralis, i.e. "sustained" cells which provide the basis for spatial discrimination, but not in "transient" neurones in the peripheral retina.

VON NOORDEN We have done this and the foveal and peripheral areas do not seem to differ in any way from the normal, unfortunately for your theory.

HAWORTH Can any estimate be made of the age at which the ganglion cells develop their connections in humans?

IKEDA It would probably coincide with the development of a functional fovea, and we are not certain at what age this occurs, although some reports suggest that it is within the first few months of life.

WYBAR The fovea is well developed at birth. Apparently the poor vision of very young children is due to lack of attention rather than to the structure of the eye. Further development is due to an increase in neuronal connections and not to any structural change in the eye.

JARDINE The concept of the degeneration of ganglion cells in amblyopia seems doubtful, since a child with an amblyopia of 6/6o can frequently be brought up to a visual acuity of $6 / 6$ within a few weeks. I feel that the ganglion cell could not possibly be regenerated in this short period of time.

* The film was shown at the meeting 
VON NOORDEN Chow and Stewart (1972) showed that, in animals which were deprived of visual stimuli to one eye for a period of time and then allowed to see normally again, the ganglion cells which had previously been small had expanded again to their normal size. It is probable, therefore, that if there is a type of disuse atrophy of the ganglion cells and they are stimulated again at a later time, these cells would be restored to their normal size. If, however, the ganglion cell had never received any stimulus, then such changes would, of course, be irreversible.

BLAKEMORE It is most important to get away from the idea that cell size is correlated with cell function. One must look at the synaptic structure. Enormous changes in cell function can occur without any change in cell size, but certainly neuronal connections can be made or unmade, and this might account for the observations.

BAGOLINI I should expect a less out-of-focus image at the fovea of the deviating eye when the angle of deviation is small and a more grossly out-of-focus foveal image when the angle is large. If Dr. Ikeda's hypothesis is correct, a deeper amblyopia should then be expected in large-angle than in small-angle strabismus, which does not coincide with clinical experience.

LYLE I was certain that a large number of people with small-angle squint became more densely amblyopic than those with large-angle squint.

IKEDA We have examined the literature and, although we have heard that this was indeed so, we have never found any report in which this could be shown to be a fact. At the moment I consider it is unproven. The idea we have put forward is that if a child receives a blurred image at the fovea or near the fovea for whatever reason (whether from an isometropia, hypermetropia, opacity in the media, or a squint) in the early sensitive period of development, then amblyopia will result. We should have to look into what really happens to the foveal image when the angle of squint is small and compare this with the foveal image of a large-angle squint, before we could answer the question.

GILKES It is surprising that an increase in the refractive error produced a proportional response in the reduction of retinal sensitivity, as clinical evidence suggests that there is no correlation between the amount of refractive error and the density of the amblyopia.

ARDEN Dr. Ikeda's Figure is based on, of course, the laws of physics applied to the eye, i.e. progressive defocusing produces progressive image spread on the retina. The fact that you do not see less with severe defocusing than with moderate defocusing is a very powerful argument in favour of Dr. Ikeda's theory in man.

HAWORTH If a child is born with bilateral hypermetropia, is it likely to be amblyopic as compared with a normal child?

IKEDA Yes, according to our hypothesis, both eyes are likely to become amblyopic.

REINHART Would it be reasonable to characterize your theory as primarily an input theory? An amblyopia as the result of degeneration of the input element? I notice that you do allow for active suppression. Do you have any data to support this, in addition to your input data?

IKEDA It is almost impossible experimentally to show active suppression of a perfect message arising from the retina at the cortex. The two theories, inadequate input and active suppression, are not at all incompatible. The inadequate input theory may explain the reduction of visual acuity of the fovea in cases in which the fovea was deprived of adequate stimulation in early development, while active suppression may also occur at the cortical level so that binocular vision is disturbed in squint amblyopia.

BAGOLINI What Dr. Ikeda says about suppression may have some support from the literature (Bagolini; Maraini and Pasino; Halldén). There appear to be two antidiplopic mechanisms of amblyopia which are seen in patients examined in when merely looking without introducing artefacts. The first is the supression which occurs in the binocular visual field of the deviating eye mainly in large-angle strabismus. The other is anomalous correspondence which appears to prevail in small- 
angle deviation. Amblyopia may be present in anomalous retinal correspondence in spite of the fact that suppression plays a doubtful role, and seems to be related to an inhibition of normal retinal correspondence. True suppression scotomata are not present. An anomalous pattern is facilitated, leading to a sort of anomalous binocular vision with an anomalous overlapping of the two binocular visual fields. It seems to me that Dr. Ikeda's experimental findings support these new points of view about amblyopia.

HAWORTH There must be separate mechanisms of amblyopia. In an anisometropic individual one retinal image would be always blurred which might account for the amblyopia. In a squinting eye, however, the foveal image might be perfectly sharp and the amblyopia more likely to be due to active suppression. Also, the angle of squint should not affect the degree of amblyopia whatever the mechanism involved.

IKEDA I agree. I have been talking about blurring of the foveal images, and not blurring of the eccentric image, because on this point $I$ have considered that amblyopia means a reduction of visual acuity of the fovea.

SANDERS What is the role of colour vision in the sustained cells? In my clinical experience, one clearly sees amblyopes who have reduced visual acuity, but normal colour vision. Are the sustained cells not functioning as colour transmitters?

IKEDA I cannot answer this question because we have not studied the colour-coded retinal ganglion cells or cortical cells. To study colour while controlling for luminance is exceptionally difficult, and we do not have an adequate set-up for this. But it has been shown by Dr. Gouras in the United States that some sustained cells in the area centralis are colour-coded. We found that some sustained firing cortical neurones were not tuned sharply to orientation but that they might be tuned to colour, although I have no firm evidence that this was or was not so. If squinting eyes were deprived of colour as well as sharply focused small objects in early development, one might predict that colour vision as well as visual acuity would be affected. But if the deprivation consists of loss of a welldefined image as we propose, colour vision may be normal while the visual acuity may be reduced, because colour-coded sustained retinal ganglion cells may not be deprived of adequate stimulation.

PARKS Has any experimentation been undertaken simultaneously on both eyes? I had the impression that all the experimentation had been done on one eye only. I wondered if binocular experiments had been performed on similar regions of both retinae and whether any difference was observed in cortical responses between one eye and the other.

IKEDA Yes, it is important to do this, but so far our experiments have been done only on normal cats, and in these experiments corresponding retinal areas of the two eyes behave similarly. In fact, experiments as described by Dr. Parks had been planned in our amblyopic animals, but unfortunately all our cats died at a crucial moment, and this made it impossible for us to test our theory.

HARCOURT Has the fact that the sustained cells do not respond to fast moving targets any signicance in our attempt to assess visual acuity using optokinetic moving stimuli?

IKEDA This appears to be dependent on the speed of rotation of the drum. In order to stimulate the sustained system a slow rotation of fine grating seems best.

VAN SLUYTERS We have tested the visual behaviour of our cats and we have not found any signs of amblyopia affecting the visual behaviour and I am intrigued to see that Dr. Ikeda has found these changes.

IKEDA We have made a film showing definite behavioural difficulties in cats reared with a surgical squint in one eye.* The animals behaved normally when both eyes were open, but if the squinting eye was the only one in use (the normal eye being covered by an opaque lens) the cat was unsure of himself, unable to pick up small objects, and unable to judge distances. These kittens were retarded compared with normal animals in their visual exploratory behaviour. 